\title{
CEREBRAL AIR EMBOLISM: A NON-THROMBOTIC CAUSE OF STROKE
}

Martina Squitieri *, MD, Francesca Pescini ${ }^{* *}, \mathrm{MD}, \mathrm{PhD}$; Anna Poggesi*, MD, PhD; Leonardo Pantoni ${ }^{\circ}, \mathrm{MD}, \mathrm{PhD}$.

* NEUROFARBA Department, Neuroscience Section, University of Florence, Florence, Italy

** Stroke Unit, Azienda Ospedaliero Universitaria Careggi, Florence, Italy

○ "L. Sacco" Department of Biomedical and Clinical Sciences, University of Milan, Milan, Italy

\section{INTRODUCTION}

The majority of ischemic strokes are of an embolic nature and are commonly due to occlusion of the brain arteries by clots. Less frequently, emboli may consist of non-thrombotic material.

Cerebral air embolism (CAE) is a rare cause of non-thrombotic stroke. It may occur as complication of medical procedures, although non-iatrogenic sources have been also reported.

\section{Patient 1}

A 71-year-old man underwent a coronary artery bypass grafting. 20 days later, soon after a CVC manipulation, he suddenly presented loss of consciousness, abnormal flexion response to pain, no verbal response (Glasgow Coma Scale-GCS: 5). Brain CT (fig.1) and brain MRI (fig.2) were performed. Angio-CT did not show occlusions of the main cerebral vessels. He developed epileptic focal status that was a contraindication for hyperbaric oxygen therapy. Patent foramen ovale (PFO) was excluded. The patient developed an unresponsive wakefulness syndrome.

Fig.3. Brain CT: multiple hypodensities in the sulci of right temporooccipital area.

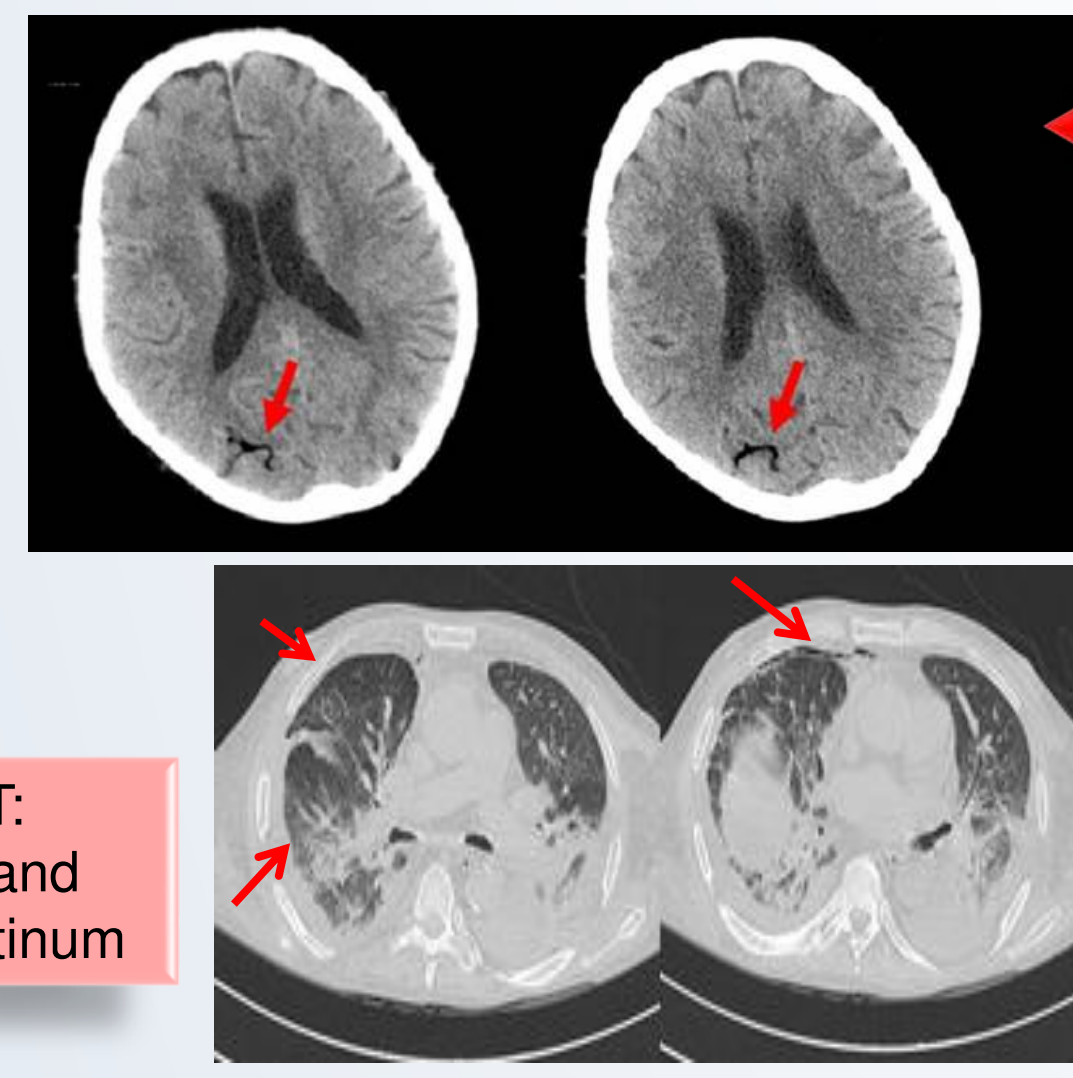

Fig.4. Chest CT: Pneumothorax and pneumomediastinum
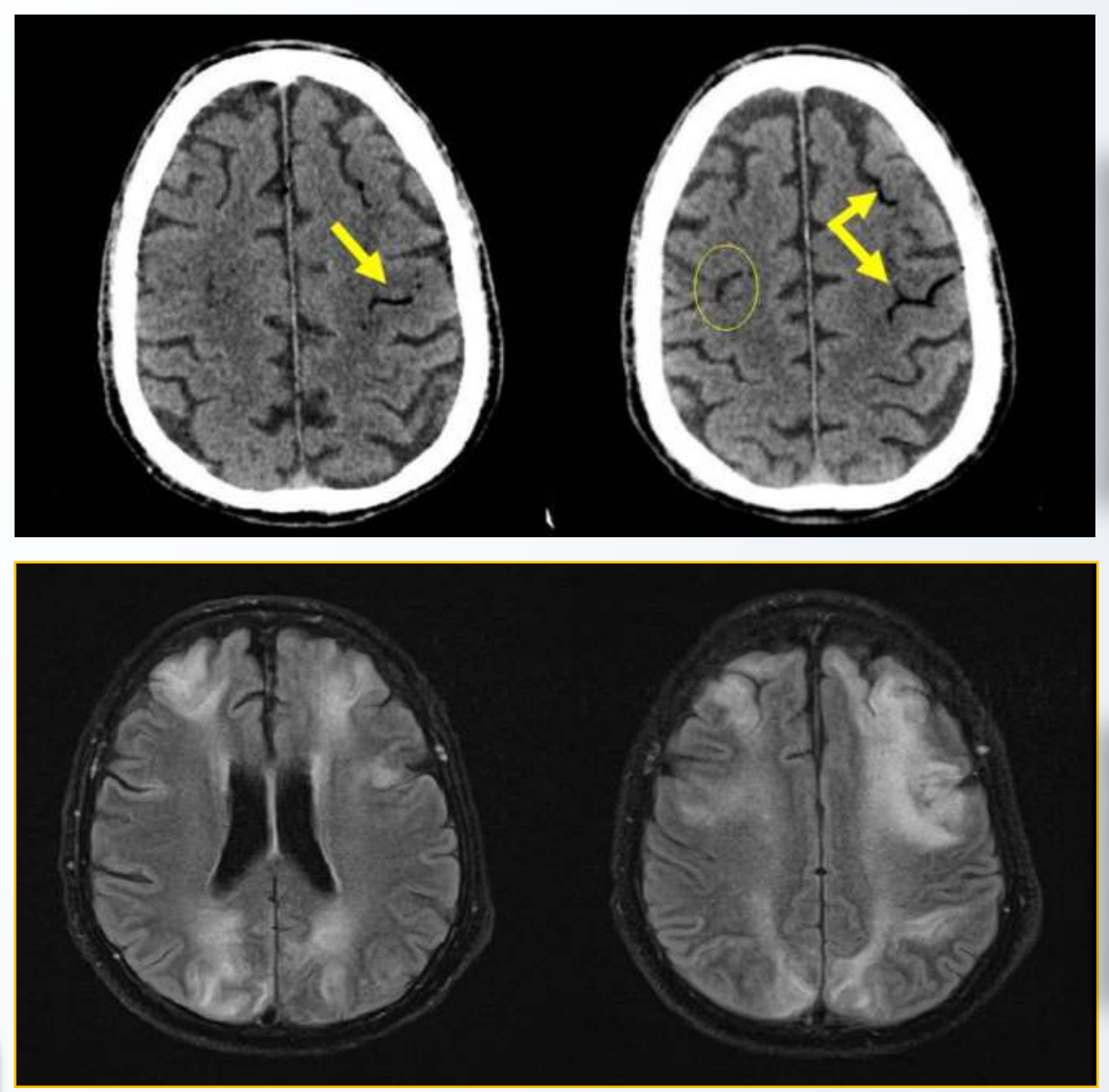

Fig.1. Brain CT: multiple hypodensities in the sulci of left parietal and right frontal lobe

Fig.2. Brain MRI: multiple bilateral cortical ischemic lesions

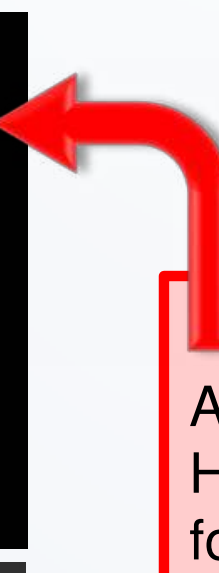

A $71-y$

Patient 2

A 71-year-old male performed a coronary angiography for heart failure. He had end-stage renal disease and experienced pulmonary lobectomy for a cystic lesion. On admission, pneumonia was diagnosed. On day 2, he presented prolonged coughing and acute respiratory distress. After few minutes he became suddenly unresponsive with head and eyes deviation to the left side (GCS 7). Neurological examination showed: no eye opening, orientated verbal response, left arm plegia and paresis of the legs (NIHSS 16). 30 minutes later, brain CT (fig.3) and chest CT (fig.4) were performed. Pneumothorax and pneumomediastinum were detected so hyperbaric oxygen therapy was not applied.

\section{Patient 3}

A 84-years-old woman was admitted to the Cardiosurgery Department for an aortic valve replacement. 1 hour after CVC removing, she presented right leg clonic jerks and right deviation of head and eyes. After 10 minutes a complete neurological examination showed: no verbal response, right arm plegia, legs and left arm paresis, complete right hemianopia, severe sensory loss in the right hemisoma and right facial palsy. Bilateral Babinski sign was found (NIHSS 32). Brain CT was performed 20 minutes later (fig.5); angio-CT did not show occlusions of the main cerebral vessels.

She developed an allergic response to contrast media and she needed non invasive ventilation. Bronchospasm with elevated $\mathrm{CO} 2$ levels in the blood was a contraindication for hyperbaric oxygen therapy.

\section{DISCUSSION}

CAE may occur during CVC placement, removal or manipulation and also consequently to non-iatrogenic conditions such as pulmonary diseases, even if this latter is rare.

Clinical manifestations are similar to those of thrombotic ischemic strokes, although some features such as decreased consciousness and seizures are more frequent in CAE.

Brain CT is useful to detect cerebral air bubbles when performed in the hyperacute phase. Hyperbaric oxygen therapy is the gold standard treatment for CAE within the first hours (up to 6) if contraindications are not present.

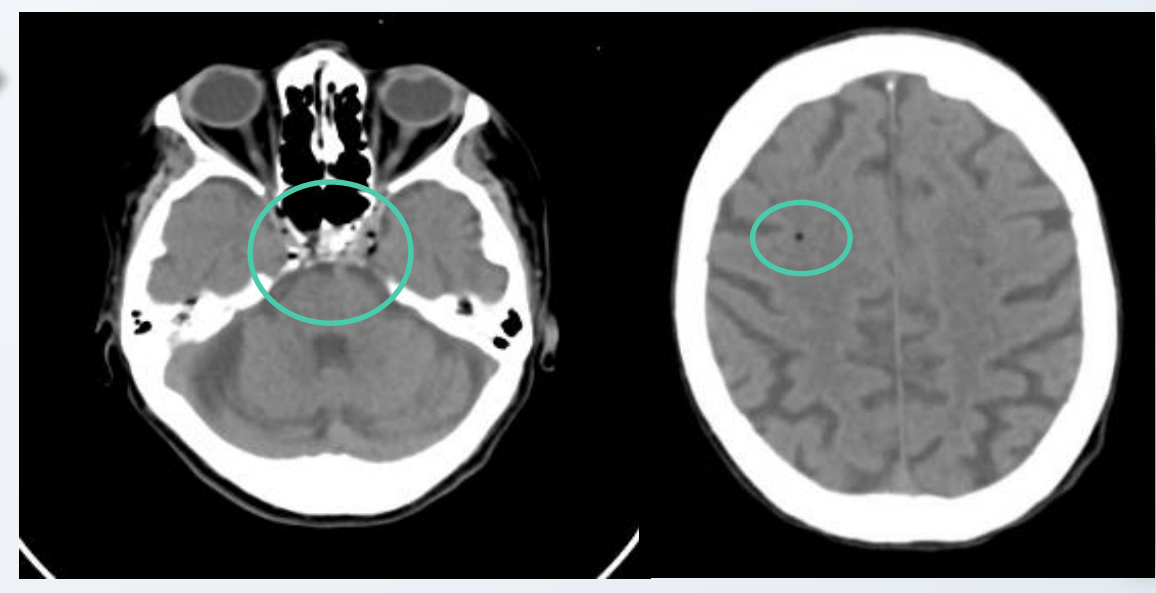

Fig.5. Brain CT: multiple hypodensities in the sulci of right frontal lobe and in both cavernous sinus.

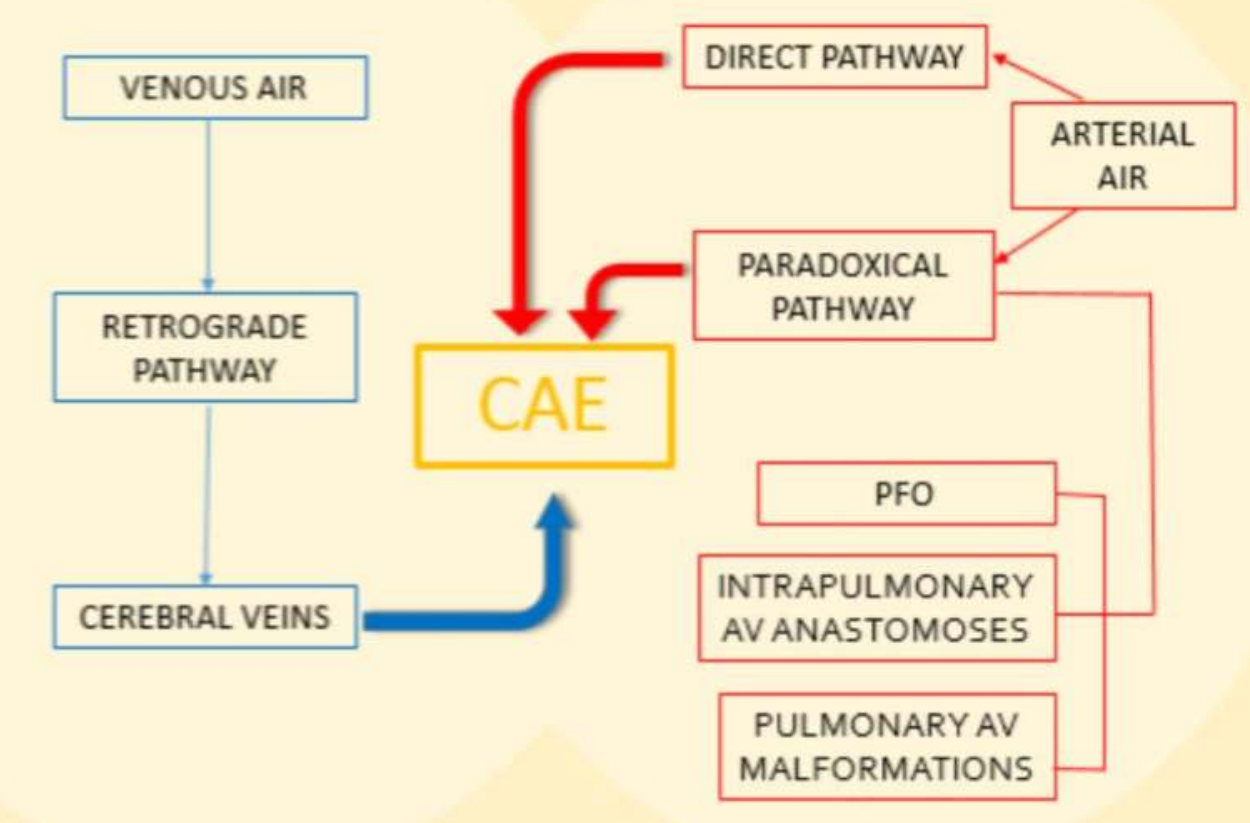

REFERENCES

Judge C. et all, Stroke Research and Treatment. 2017; 2017:7565-702 Pinho J. et all, Journal of the Neurological Sciences, 362; 160-164, 2016. 